\title{
Improving the detection of rare native fish species in environmental DNA metabarcoding surveys
}

\author{
Jack Rojahn‡, Dianne M Gleeson‡, Elise Furlan‡, Tim Haeusler§, Jonas Bylemans \\ ‡ University of Canberra, Canberra, Australia \\ $\S$ New South Wales department of Planning, Industry and Environment, Wollongong, Australia \\ | University of Lausanne, Lausanne, Switzerland
}

Corresponding author: Jonas Bylemans (jonas.bylemans@unil.ch)

Received: 02 Mar 2021 | Published: 04 Mar 2021

Citation: Rojahn J, Gleeson DM, Furlan E, Haeusler T, Bylemans J (2021) Improving the detection of rare native fish species in environmental DNA metabarcoding surveys. ARPHA Conference Abstracts 4: e65387.

https://doi.org/10.3897/aca.4.e65387

\begin{abstract}
The presence of threatened or endangered species often strongly influences management and conservation decisions. Within the Murray-Darling Basin (MDB), Australia, the presence of threatened native fish affects the management and allocation of water resources. These decisions are currently based on traditional fisheries data and a predictive MaxEnt model. However, it is important to verify the model's predictive power given the implication it may have, but this requires methods with a high detection sensitivity for rare species. Although the use of environmental DNA (eDNA) metabarcoding achieves a higher detection sensitivity compared with traditional methods, earlier surveys in the MDB have shown that the highly abundant and invasive common carp (Cyprinus carpio) can reduce detection probabilities for rare species. Consequently, a polymerase chain reaction (PCR) blocking primer designed to block the amplification of carp eDNA could increase the detection probabilities for rare native species while simultaneously reducing the required sampling effort and survey costs. Although PCR blocking primers are often used in ancient DNA and dietary studies, no aquatic eDNA metabarcoding study to date has evaluated the potential benefits of using PCR blocking primers. A laboratory and fieldbased pilot study was used to address this knowledge gap and assess the impact of a blocking primer on the detection probabilities of native species and the minimum sampling effort required. Results showed that the inclusion of the blocking primer increased the detection probabilities for native species by $10-20 \%$ and reduced the minimum required
\end{abstract}


sampling effort by $25-50 \%$. These findings provide important insights into possible methods for optimizing eDNA metabarcoding surveys for the detection of rare aquatic species.

\section{Keywords}

environmental DNA, metabarcoding, blocking primer, fishes

\section{Presenting author}

Jonas Bylemans

\section{Presented at}

1st DNAQUA International Conference (March 9-11, 2021) 